University of Wollongong

Research Online

Faculty of Business - Papers (Archive)

Faculty of Business and Law

$1-1-2013$

Environmental shocks and sustainability in a basic economy-environment model

Fabio Privileggi

Universita Del Piemonte Orientale

Simone Marsiglio

University of Wollongong, simonem@uow.edu.au

Follow this and additional works at: https://ro.uow.edu.au/buspapers

Part of the Business Commons

Research Online is the open access institutional repository for the University of Wollongong. For further information contact the UOW Library: research-pubs@uow.edu.au 


\title{
Environmental shocks and sustainability in a basic economy-environment model
}

\begin{abstract}
We study a stochastic, discrete-time, economy-environment integrated model, where human activity affects the evolution of pollution over time. We assume that exogenous i.i.d. environmental shocks determine the rate of pollution transfer. We show that the pollution to capital ratio dynamics can be read as an iterated function system converging to an invariant distribution supported on a (asymmetric) Cantor set, and that human intervention aiming at offsetting the environmental impact of economic activities is needed to ensure sustainability.
\end{abstract}

Keywords

economy, environment, model, environmental, sustainability, shocks, basic

Disciplines

Business

Publication Details

Privileggi, F. \& Marsiglio, S. (2013). Environmental shocks and sustainability in a basic economyenvironment model. International Journal of Applied Nonlinear Science, 1 (1), 67-75. 


\title{
Environmental shocks and sustainability in a basic economy-environment model
}

\begin{abstract}
We study a stochastic, discrete-time, economy-environment integrated model, where human activity affects the evolution of pollution over time. We assume that exogenous i.i.d. environmental shocks determine the rate of pollution transfer. We show that the pollution to capital ratio dynamics can be read as an Iterated Function System converging to an invariant distribution supported on a (asymmetric) Cantor set, and that human intervention aiming at offsetting the environmental impact of economic activities is needed to ensure sustainability.
\end{abstract}

Keywords: Iterated Function System, Cantor Set, Environmental Shocks, EconomyEnvironment Model, Sustainability

JEL classification: C61, O41, Q50

\section{Introduction}

It is by now well known that economic systems may exhibit complex dynamics through time, while simple stochastic models can even portrait a fractal structure when the number of exogenous shocks is finite. The purpose of the present work is to widen this family of models to those envisaging environmental consequences of human economic activity. Recently, the analysis of anthropogenic degradation of the environment has obtained raising interest in social sciences because of its implications on the wellbeing of current and future generations. We therefore present a very simple integrated model of the economy and environment in order to exemplify the possible effects of random shocks on both the economic and environmental variables. In particular, we assume that humans affect the environment through production activities by generating pollutants and the environment itself is subject to random shocks determining its rate of pollution absorption. We show that in such a framework where humans do not intervene (not even partially) to clean up the environment, at equilibrium it is more likely ending up in a situation of high pollution, which is clearly unsustainable. If we want to improve such an outcome, specific interventions are needed in order to preserve the environment.

Mandelbrot (1982), in his seminal work, firstly introduced the notion of fractals, namely sets that may be expressed as unions of contracted copies of themselves (self-similarity) and have complex geometric and topological patterns (e.g., they can be perfect and totally disconnected), along with several examples. The Cantor set is probably the most well known example of such sets. Hutchinson (1981), Barnsley and Demko (1985) and Barnsley (1989) then showed that Iterated Function Systems (IFS), that is, systems of contractive maps with associated probabilities, can be used to construct fractals, self-similar sets and measures supported on such sets. These sets and measures are attractive fixed points of fractal transform operators. A wide theory on IFS has been developed lately (see La Torre and Vrscay, 2011, for a brief 
survey) and applications of this theory in several fields, among which even economics, have been widely developed.

Boldrin and Montrucchio (1986) firstly showed that even in the simplest deterministic concave intertemporal economic model complicated optimal dynamics can occur. Their result gave birth to a large literature with the goal of studying complexity and chaos in economic models. A consistent part of this literature focussed on stochastic concave intertemporal models, as in the Brock and Mirman (1972) tradition. Montrucchio and Privileggi (1999), by using the Collage Theorem (Hutchinson, 1981; Barnsley, 1989; Vrscay, 1991), showed that optimal dynamics in standard stochastic concave growth models are random processes that can converge to singular invariant distributions supported on some fractal sets. Mitra et al. (2003) analyzed a one-sector growth model with two random shocks whose optimal trajectory may converge to a singular distribution supported on a Cantor set, also characterizing singularity versus absolute continuity of the invariant probability measure. Mitra and Privileggi (2004, 2006, 2009) further generalized that result by establishing conditions on the fundamentals under which the invariant probability happens to be supported on a Cantor set, and provided an estimate of the Lipschitz constant for the optimal policy. La Torre et al. (2011) and Marsiglio (2012) showed that the optimal path of a two-sector growth model subject to random shocks may converge to an invariant distribution supported on some fractal set.

The paper proceeds as follows. Section 2 describes our simple economic-environmental framework, and shows that the pollution to capital ratio can be read as an IFS converging to an invariant distribution supported on an 'asymmetric' Cantor set. Section 3 develops a specific example more thoroughly and proposes an interpretation of the results in terms of sustainability. Section 4 contains concluding remarks.

\section{The model}

We study the simplest ${ }^{1}$ discrete time economy-environment integrated model under uncertainty in which the economic side affects the environmental side via the emission of pollutants, and the environment itself is affected by random shocks determining the rate of natural pollution absorption. As in Solow (1956), economic activity employs capital, $k_{t}$, in order to produce a unique homogenous good, $y_{t}$, which is exogenously allocated to consumption and investment activities. In particular, the share of output devoted to consumption needs is $1-s$ and the remaining share, $s$, is devoted to investments in capital accumulation, where $0<s<1$ is the saving rate. Output is produced according to a linear production function employing only capital: $y_{t}=A k_{t}$, where $A$ is a scale parameter representing technical knowledge. As a result the economy is simply represented by the evolution of capital over time: $k_{t+1}=s y_{t}=s A k_{t}$.

Economic production generates the emission of pollutants which negatively affect the evolution of environmental quality over time. Emissions are a side product of economic activity and are assumed to be proportional to output, $\eta y_{t}$, where $\eta>0$ is a technological parameter determining environmental efficiency. Pollution, $p_{t}$, has a rate of time decay equal to $\theta_{t}, 0<\theta_{t}<1$, such that its evolution over time is given by the sum of anthropogenic and natural pollution: $p_{t+1}=\left(1-\theta_{t}\right) p_{t}+\eta y_{t}$, or equivalently: $p_{t+1}=\delta_{t} p_{t}+\eta A k_{t}$, where $\delta_{t}=1-\theta_{t}$ represents net (of absorption capacity) transfer of pollution from one period to the next.

\footnotetext{
${ }^{1}$ In order to keep the model as simple as possible, we abstract from agents' optimal behavior and no consumption and endogenous allocation of resources is taken into account. However, the introduction of agents with optimizing behavior will not lead to qualitatively different results (compare for example the model outcomes in La Torre et al., 2011, and Marsiglio, 2012).
} 
We assume that the environment is affected by exogenous shocks which affect its rate of natural absorption, $\theta_{t}$, or equivalently its net rate of transfer, $\delta_{t}=1-\theta_{t}$. Such shocks are assumed to be independent and identically distributed, and take on two values, such that $\delta_{t} \in\left\{\delta_{\ell}, \delta_{u}\right\}$, with $0<\delta_{\ell}<\delta_{u}<1$. These two different shock configurations represent how the environment is affected by exogenous factors: external factors, as climate change or alterations in the biodiversity mix, may have a particularly large impact (if $\delta_{t}=\delta_{u}$ ) or a moderate effect (if $\delta_{t}=\delta_{\ell}$ ) on the pollution transfer rate. The economy-environment dynamics can be therefore summarized by the following planar system of linear difference equations:

$$
\begin{aligned}
& k_{t+1}=s A k_{t} \\
& p_{t+1}=\delta_{t} p_{t}+\eta A k_{t},
\end{aligned}
$$

with $\delta_{t} \in\left\{\delta_{\ell}, \delta_{u}\right\}$.

Equation (1) underlines the importance of capital accumulation in driving the evolution of industrialized economies in the long-run; this is a very well known issue in the economic literature since Solow's (1956) seminal work. Equation (2) says that capital does not have only positive effects on the well being of modern societies but does also have a negative impact, through the increasing emissions generated by increasing output and its consequences on health and other aspects related to happiness (not considered in our simplified framework). Therefore, as a society gets richer and richer (capital increases) it becomes also more and more stringent the need to understand how avoiding dramatic environmental changes (increases in pollution). This is the main goal of the sustainability ${ }^{2}$ literature, which wonders whether and how a certain level of environmental quality has to be preserved (World Commission on Environment and Development, 1987).

It is straightforward to verify that the system $(1)-(2)$ is diverging, since both capital and pollution grow without any bound. Therefore, in such a framework we need to define a steady state as a situation in which some other variable is converging to a stationary equilibrium. The natural candidate seems to be the pollution to capital ratio, $\chi_{t}=p_{t} / k_{t}$. In fact, if this new variable converges to a finite value, this will mean that the original variables $\left(p_{t}\right.$ and $\left.k_{t}\right)$ are growing at the same rate; such a situation can be directly interpreted as a balanced growth path equilibrium. Note that the pollution to capital ratio evolves over time according to a linear difference equation too:

$$
\chi_{t+1}=\phi\left(\chi_{t}\right)=\frac{\delta_{t}}{s A} \chi_{t}+\frac{\eta}{s} .
$$

It is straightforward to see that if $\delta_{u} / s A<1$ the map $\phi\left(\chi_{t}\right)$ is contractive, and therefore the random dynamical system (3) converges to an invariant distribution. From now onward, we will assume that such a condition is verified. Recalling the results of Hutchinson (1981) and Barnsley et al. (2008), it is straightforward to prove that (3) defines an IFS converging to an invariant distribution supported on an asymmetric Cantor set whenever a no-overlap condition is verified.

Proposition 1 Assume that the following no-overlap condition is satisfied:

$$
s A>\delta_{u}+\delta_{\ell} ;
$$

\footnotetext{
${ }^{2}$ The Brundtland Commission firstly defined sustainable development as that type of development that "satisfies the needs of the present without compromising the ability of future generations to meet their own needs" (World Commission on Environment and Development, 1987). Sustainability is therefore related to two distinct concepts, respect of natural resources and intergenerational equity (see Marsiglio, 2011, for further details).
} 
then the pollution to capital ratio, as in (3), defines a contractive linear IFS composed of two linear maps $\phi_{0}, \phi_{1}:[a, b] \rightarrow[a, b]$, where $a=\eta A /\left(s A-\delta_{\ell}\right)$ and $b=\eta A /\left(s A-\delta_{u}\right)$ are the fixed points of $\phi_{0}$ and $\phi_{1}$ respectively, given by:

$$
\begin{cases}\phi_{1}\left(\chi_{t}\right)=\frac{\delta_{u}}{s A} \chi_{t}+\frac{\eta}{s} & \text { with probability } p \\ \phi_{0}\left(\chi_{t}\right)=\frac{\delta_{\ell}}{s A} \chi_{t}+\frac{\eta}{s} & \text { with probability } 1-p\end{cases}
$$

Such an IFS converges weakly to a unique invariant distribution supported on an asymmetric Cantor set.

Proof. Clearly the no-overlap condition (4) implies $\delta_{u} / s A<1$, so that the IFS (5) is a contraction. Therefore, the theory on IFS, as developed in Hutchinson (1981) and Barnsley et al. (2008) applies directly to establish weak convergence of the IFS to a unique invariant probability supported on a subset of $[a, b]$.

Moreover, the no-overlap condition (4) ensures that $\max _{[a, b]} \phi_{0}(\chi)<\min _{[a, b]} \phi_{1}(\chi)$, so that the two image sets $\phi_{0}([a, b])$ and $\phi_{1}([a, b])$ do not overlap; in fact, as both $\phi_{0}$ and $\phi_{1}$ are monotone increasing, this is equivalent to $\phi_{0}(b)<\phi_{1}(a)$, which, after some rearranging, leads to $\left(s A-\delta_{u}-\delta_{\ell}\right)\left(\delta_{u}-\delta_{\ell}\right)>0$, which, as $\left(\delta_{u}-\delta_{\ell}\right)>0$, is equivalent to (4). Hence, at each iteration of the IFS (5) a hole appear in all components of the pre-fractal, so that in the limit a Cantor-like set appears as unique invariant set for the system; see, e.g., Falconer (1997, 2003), Mitra et al. (2003), and Mitra and Privileggi (2004, 2006, 2009) for details.

By asymmetric Cantor set here we mean a Cantor-like set in which the components in the left tail are smaller than those in the right tail; this is due to the different slopes of the linear maps $\phi_{i}$ in (5), the slope of the lower map $\phi_{0}$ being smaller than that of the upper map $\phi_{1}$, as illustrated in Figure 1 further on.

Note that the required no-overlap (and thus contraction) condition, $\delta_{u}+\delta_{\ell}<s A$, is verified if the rates of pollution transfer are not too large. Empirical estimates of $s$, the saving rate, and the benchmark value of $A$, the productivity index, in industrialized economies are around 0.5 (see Mankiw et al., 1992) and 1 (see Mulligan and Sala-i-Martin, 1993) respectively. This means that the pollution rates of transfer have to be bounded from above, specifically, their sum must satisfy $\delta_{u}+\delta_{\ell}<0.5$ in order to meet the requirements in Proposition 1 . Whether this condition is verified or not can vary according to the type (air, soil or water pollution) of pollutants we are considering, thus it has only to be interpreted as a technical condition.

Our result in Proposition 1, giving conditions under which a discrete time economic model subject to random shocks converges to an invariant distribution supported on an asymmetric Cantor set, is very similar to that presented in Marsiglio (2012). Marsiglio (2012) shows that the capital ratio (the ratio between two different types of capital, both employed in the production of a unique final good) converges to an invariant distribution and the presence of marginal returns in both the capital sectors ensures such a convergence. The peculiarities of our result are two: (i) we show that in economic-environmental models the pollution to capital ratio may converge to a fractal set under a suitable choice for the parameters' values; and (ii) we confirm again the well known fact that very simple linear models - see equations (1) and (2) - may exhibit such non-trivial dynamics. 


\section{A numerical example}

Figure 1 shows the first three iterations of the IFS in (5) for f $^{3} \eta=s=0.5$ (so that $\eta / s=1$ ), $A=1$ (so that $s A=0.5$ ), $\delta_{\ell}=0.11$ and $\delta_{u}=0.25$ starting from the initial set $[a, b]$ in $t=0$, with $a=\eta A /\left(s A-\delta_{\ell}\right)=1.282$ and $b=\eta A /\left(s A-\delta_{u}\right)=2$, a set of parameters' values that clearly satisfy the assumptions of Proposition 1 . Because the slope of $\phi_{1}$ is larger than that of $\phi_{0}$, it can be seen that each iteration yields a union of intervals of increasing length when moving from $a$ to $b$, while the middle pairs of (groups of) intervals have the same size, as can be seen on the vertical axis in Figures 1(a) and 1(b). As a result, each pre-fractal replicates the asymmetry in the slopes of the two maps $\phi_{i}$, containing intervals of different lengths, distributing some of them with the same length around the middle of $[a, b]$ while filling the tail close to $a$ with intervals smaller than those close to $b$, as can be grasped from Figure 1(c).

Even without considering the probability $p$ attached to $\phi_{1}$ - the worst scenario characterized by the largest pollution transfer $\delta_{u}$ - in the IFS (5), from Figure 1 it is striking the impact of the asymmetry of the Cantor-like set on the long-run outcome of our economic-environmental system in terms of possible states of nature. ${ }^{4}$ Since the components in the left tail are smaller than those in the right tail, we have a clear imbalance of the pollution to capital ratio towards a high pollution situation. This means that in our benchmark framework, where human activity is uniquely oriented toward economic growth in a production process that generates pollutants as side-effect and green interventions (actions devoted to reduce the human impact on the environment) are not undertaken, at equilibrium future generations will have to bear larger environmental costs. ${ }^{5}$ Clearly, a small (exogenous) probability $p$ associated to the "bad state" $\phi_{1}$ would mitigate such costs; however, whenever $p$ is large enough, such a scenario clearly becomes not sustainable, as it contrasts with the concept of intergenerational equity (World Commission on Environment and Development, 1987). Note also that in a more realistic model it is likely that human activity affects the probability $p$ as well, causing it to increase as industrial production intensifies.

All these considerations lead to the conclusion that in order ensure sustainability some efforts to preserve the environment are needed. Moreover, as we can see from Figure 1, since $a>0$, a positive amount of pollution will always be present in any state of the world. When social welfare depends strongly on environmental quality, it will be highly desirable to reach a zero pollution situation; this is clearly not possible without purposive green interventions.

\footnotetext{
${ }^{3}$ The empirical values of $\eta$, environmental efficiency in economic production activity, is highly uncertain and varies dramatically according to the type of pollution we are considering. Since in our simulation, its value does not affect the qualitative results, for sake of simplicity we set it equal to the saving rate.

${ }^{4}$ Recall that the Cantor set supports the invariant probability distribution of the system.

${ }^{5}$ Note that the model abstracts completely from any notion of welfare and any distinction between current and future generations. The interpretation of the results we are presenting here rely on the fact that our dynamic model represents a simplification of a model where agents optimize their behavior. In particular, we should think of a social planner wishing to maximize the social welfare given the law of motion of capital and pollution, as in the Ramsey (1928) tradition. We leave this project for future research.
} 


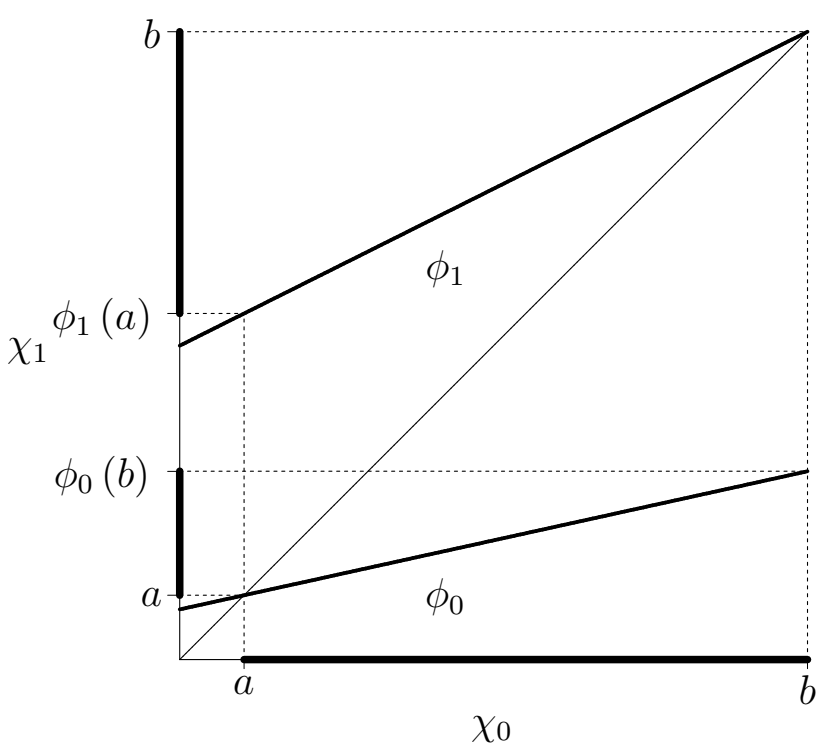

(a)

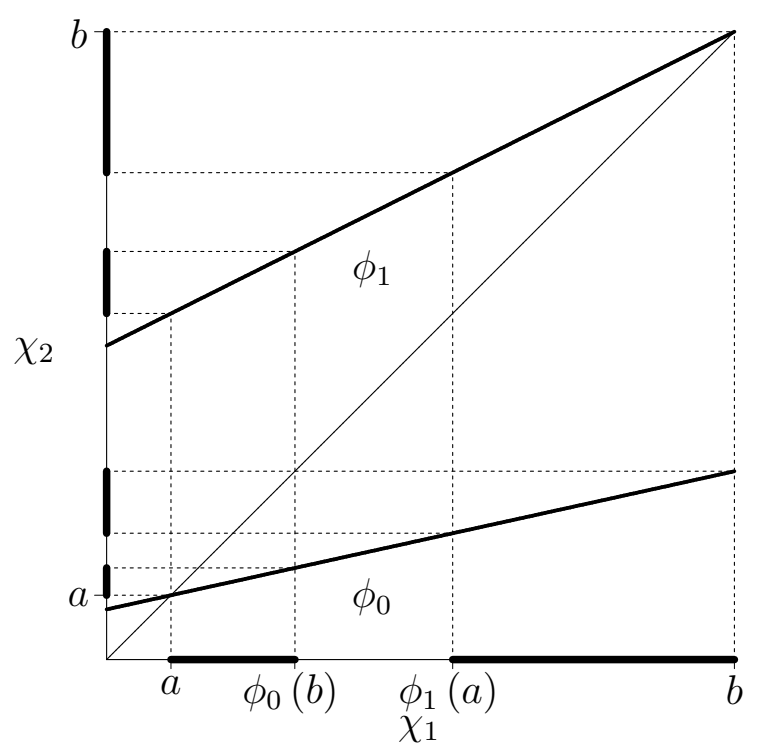

(b)

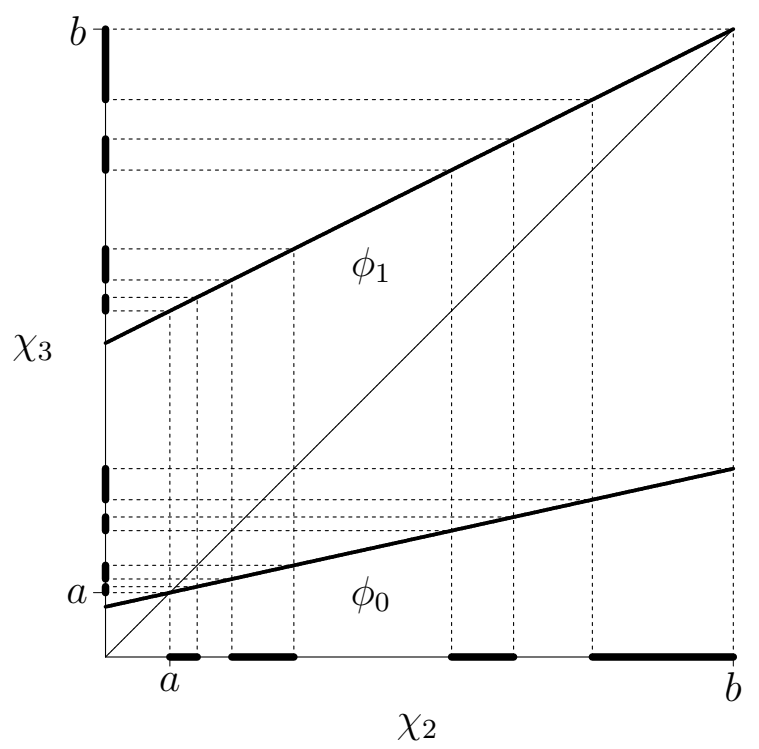

(c)

FIgURE 1: first three iterations of the IFS in (5) for $\eta=s=0.5, A=1, \delta_{\ell}=0.11$ and $\delta_{u}=0.25$ starting from the initial set $[a, b]$ in $t=0$, with $a=1.282$ and $b=2$.

\section{Conclusions}

We studied a very simple economic-environmental model in which the economic side affects the environmental side via the emission of pollutants, showing that the pollution to capital ratio dynamic equation is simply an IFS converging to an invariant distribution supported on an asymmetric Cantor set. This is a very simple example illustrating how an integrated economyenvironment model affected by random shocks can be read as an IFS, and this allows us to characterize directly its long-run properties. The asymmetry in our Cantor-like set clearly implies an imbalance towards a high pollution situation, which results to be far away from any notion of sustainability. In order to improve such an outcome, some green interventions (switching from high to low polluting technologies or devoting specific resources to clean up the 
environment) are needed. Sustainability is a complicated issue which, as showed by our simple example, does not have to be taken for granted. Clearly, a social regulator would play a crucial role in this process.

\section{References}

[1] Barnsley MF (1989). Fractals everywhere. New York: Academic Press.

[2] Barnsley MF, Demko S (1985). Iterated function systems and the global construction of fractals. Proc Roy Soc London Ser A 399: 243-275.

[3] Barnsley MF, Hutchinson J, Stenflo O (2008). $V$-variable fractals: fractals with partial self similarity. Advances in Math 218: 2051-2088.

[4] Boldrin M, Montrucchio L (1986). On the indeterminacy of capital accumulation paths. Journal of Economic Theory 40: 26-39.

[5] Brock WA, Mirman LJ (1972). Optimal economic growth and uncertainty: the discounted case. Journal of Economic Theory 4: 479-513.

[6] Falconer KJ (1997). Techniques in fractal geometry. New York: John Wiley \& Sons.

[7] Falconer KJ (2003). Fractal geometry: mathematical foundations and applications, $2^{\text {nd }}$ edition. Chichester: John Wiley \& Sons.

[8] Hutchinson J (1981). Fractals and self-similarity. Indiana Univ J Math 30: 713-747.

[9] La Torre D, Marsiglio S, Privileggi F (2011). Fractals and Self-Similarity in Economics: the Case of a Stochastic Two-Sector Growth Model. Image Analysis \& Stereology 30: 143-151.

[10] La Torre D, Vrscay ER (2011). Generalized fractal transforms and self-similarity: recent results and applications. Image Analysis \& Stereology 30: 63-76

[11] Mandelbrot BB (1982). The fractal geometry of nature. San Francisco: Freeman.

[12] Mankiw GN, Romer D, and Weil DN (1992). A contribution to the empirics of economic growth, Quarterly Journal of Economics 107: 407-437.

[13] Marsiglio S (2011). On the relationship between population change and sustainable development. Research in Economics 65: 353-364.

[14] Marsiglio S (2012). Stochastic shocks in a two-sector Solow model. Int J Mathematical Modelling and Numerical Optimisation 3: 313-318.

[15] Mitra T, Montrucchio L, Privileggi F (2003). The nature of the steady state in models of optimal growth under uncertainty. Economic Theory 23: 39-72.

[16] Mitra T, Privileggi F (2004). Cantor type invariant distributions in the theory of optimal growth under uncertainty. Journal of Difference Equations and Applications 10: 489-500.

[17] Mitra T, Privileggi F (2006). Cantor type attractors in stochastic growth models. Chaos, Solitons and Fractals 29: 626-637. 
[18] Mitra T, Privileggi F (2009). On Lipschitz continuity of the Iterated Function System in a stochastic optimal growth model. Journal of Mathematical Economics 45: 185-198.

[19] Montrucchio L, Privileggi F (1999). Fractal steady states in stochastic optimal control models. Annals of Operations Research 88: 183-197.

[20] Mulligan CB, Sala-i-Martin X (1993). Transitional dynamics in two-sector models of endogenous growth, Quarterly Journal of Economics 108: 739-773.

[21] Ramsey F (1928). A mathematical theory of saving. Economic Journal 38: 543-559.

[22] Solow RM (1956). A Contribution to the Theory of Economic Growth. Quarterly Journal of Economics 70: 65-94.

[23] Vrscay ER (1991). Moment and collage methods for the inverse problem of fractal construction with iterated function systems. In: Peitgen HO, Henriques JM, Penedo LF, eds. Fractals in the fundamental and applied sciences. North-Holland, 443-461.

[24] World Commission on Environment and Development (1987). Our Common Future (The Brundtland Reports). Oxford: Oxford University Press 